\title{
Estudo reológico de chocolates elaborados com diferentes cultivares de cacau (Theobroma cacao L.)
}

Rheological study of chocolates made with different cocoa (Theobroma cacao L.) varieties

\section{Autores | Authors}

\ Paula Bacelar LEITE

Universidade Federal da Bahia (UFBA) Faculdade de Farmácia Rua Barão de Jeremoabo, s/n Ondina Campus Universitário de Ondina CEP: 40170-115

Salvador/BA - Brasil e-mail: bacelarleite@yahoo.com.b

\section{Suzana Caetano da Silva LANNES Alexandre Mariani RODRIGUES Fabiana Andreia Schäfer De Martini SOARES}

Universidade do Estado de São Paulo (USP)

Faculdade de Ciências Farmacêuticas Departamento de Tecnologia São Paulo/SP - Brasil e-mail:scslan@gmail.com armariani@usp.br fabimarti@usp.br

\section{Sérgio Eduardo SOARES Eliete da Silva BISPO}

Universidade Federal da Bahia (UFBA) Departamento de Análises Bromatológicas Faculdade de Farmácia Salvador/BA - Brasil

e-mail: ssoares.ssa@gmail.com eliete.bispo@gmail.com

$\square$ Autor Correspondente / Corresponding Author

Recebido / Received: 24/01/2012 Aprovado / Approved: 14/05/2013 Publicado / Published: set./2013

\section{Resumo}

O chocolate pode ser definido como uma suspensão de partículas sólidas (açúcar, sólidos de cacau e sólidos de leite) em uma fase gordurosa contínua, que contribui para o aroma, o sabor e a cor, além de promover forma ao produto final. A reologia de chocolates é quantificada durante a produção usando-se dois parâmetros: tensão inicial (yield stress) e viscosidade aparente (plástica), em que, geralmente, se utiliza o modelo de Casson. O objetivo deste estudo foi avaliar reologicamente formulações de chocolates produzidos a partir de diferentes cultivares de cacau. As amostras de chocolate foram formuladas com $73,6 \%$ de cacau e foram realizadas as seguintes análises: determinação do teor de lipídeos; composição em triacilgliceróis; testes reológicos, e análise do tamanho máximo de partículas. O chocolate proveniente da cultivar PH16 apresentou menor conteúdo de gordura (36,53 g. $\left.100 \mathrm{~g}^{-1}\right)$, maior tamanho de partículas $(21 \mu \mathrm{m})$, maior valor de tensão inicial $(20,91 \mathrm{~Pa})$ e maior área de histerese, com mais ampla tixotropia quando comparada às amostras dos chocolates provenientes das cultivares SR162 e Convencional. Os resultados encontrados no estudo reológico mostraram a interferência do conteúdo de gordura e do tamanho de partículas na tensão inicial dos produtos.

Palavras-chave: Cacau; Chocolate; Reologia.

\section{Summary}

Chocolate can be defined as a suspension of solid particles (sugar, cocoa solids and milk solids) in a continuous fat phase, which contributes to the aroma, flavour and colour, besides giving form to the final product. The rheology of chocolates is measured during production using two parameters: the yield stress and the apparent viscosity (plastic), generally using the Casson model. The objective of this study was to rheologically evaluate chocolate formulations produced from different cocoa cultivars. The chocolate samples were formulated with $73.6 \%$ of cocoa, and analyzed for their lipid content, triacylglycerol profile, rheological tests and the maximum particle size. The chocolate obtained from the cultivar PH16 showed the lowest fat content ( $36.53 \mathrm{~g} .100 \mathrm{~g}^{-1}$ ), largest particle size $(21 \mu \mathrm{m})$, highest yield stress $(20.91 \mathrm{~Pa})$, greater hysteresis area and more ample thixotropy, when compared to samples of chocolates from the cultivars SR162 and Conventional. The results of the rheological studies showed the interference of the fat content and particle size on the yield stress of the products.

Key words: Cocoa; Chocolate; Rheology. 


\section{Introdução}

O cacaueiro (Theobroma cacao L.) é nativo da floresta tropical úmida americana, com principal origem nas nascentes dos rios Amazonas e Orinoco (GRAMACHO e MAGNO, 1992).

De acordo com o ICCO (International Cocoa Organization), os maiores produtores mundiais de cacau são a Costa do Marfim, com 1.242 mil toneladas na safra 2009/10, seguida por - também em milhares de toneladas - Gana (632), Indonésia (550), Nigéria (240), Camarões (205), Brasil (161), Equador (160) e Papua Nova Guiné (50) (ICCO, 2011).

A partir de 1989, com o primeiro relato na região cacaueira baiana do fungo Moniliophthora perniciosa, causador da doença vassoura-de-bruxa, iniciou-se nessa região um processo de empobrecimento, provocado pela redução de até $100 \%$ da produção de cacau, em diversas propriedades rurais (PEREIRA et al., 1989).

Uma das alternativas encontradas para se manejar a vassoura-de-bruxa é o emprego de variedades resistentes e de alta produtividade, desenvolvidas em programa de melhoramento genético do cacaueiro. Essa medida de controle é fundamental, tendo em vista que os controles químicos e culturais se mostram onerosos e ineficazes quando não executados rigorosamente e de acordo com as recomendações técnicas da pesquisa, além de antieconômicos, em se tratando de lavouras formadas por variedades de alta suscetibilidade e de baixa produtividade (PINTO e PIRES, 1998).

As características genéticas do cacau, bem como as operações de processamento que se iniciam na propriedade agrícola (colheita, fermentação e secagem), e as operações de processamento do chocolate - moagem do cacau, conchagem e temperagem - influenciam diretamente na qualidade final do chocolate.

Chocolate é o produto obtido a partir da mistura de derivados de cacau (Theobroma cacao L.) - massa (ou pasta ou líquor) de cacau, cacau em pó e/ou manteiga de cacau - com outros ingredientes, contendo, no mínimo, 25\% (g.100 $\left.\mathrm{g}^{-1}\right)$ de sólidos totais de cacau. O produto pode apresentar recheio, cobertura, formato e consistência variados (BRASIL, 2005). Assim, a manteiga de cacau é um dos principais ingredientes responsáveis pelo comportamento reológico de chocolates.

Reologia é o estudo da deformação e do fluxo de materiais (STEFFE, 1996). As propriedades reológicas de um material são definidas pelos seguintes aspectos: a temperatura, a pressão, a tensão e a taxa de cisalhamento. Conhecendo a magnitude desses parâmetros em processos industriais, a viscosidade e as propriedades elásticas podem ser estudadas num ambiente laboratorial, usando-se instrumentos reológicos fundamentais, tais como reômetros. Os resultados podem ser usados para estudar as diferentes propriedades dos materiais, como: estabilidade, consistência, temperatura de fusão, temperatura de endurecimento, estabilidade a tensões e peso molecular. Podem ser usados também para aperfeiçoar a qualidade de um produto e para predizer o impacto de uma formulação específica de um produto num processo industrial (MÜLLER, 1973).

A caracterização reológica de materiais - como suspensões coloidais, emulsões, espumas ou sistemas poliméricos - é complicada, pelo fato de serem viscoelásticos, ou seja, suas propriedades mecânicas estão no intervalo entre um sólido elástico e um líquido viscoso. Usando-se a reologia oscilatória, que é uma ferramenta valiosa para compreender as propriedades estruturais e dinâmicas, é possível quantificar as propriedades viscosas e elásticas de um material, em diferentes escalas temporais (STEFFE, 1996).

O objetivo deste estudo foi obter os parâmetros de Casson e de tixotropia, bem como o perfil de triacilglicerois na análise de três chocolates produzidos a partir de diferentes cultivares de cacau.

\section{Material e métodos}

\subsection{Elaboração das formulações}

\subsubsection{Material}

Foram avaliadas duas cultivares de cacau resistentes à doença vassoura-de-bruxa, denominadas de SR162 e PH16, e uma amostra de cacau convencional susceptível à doença.

A variedade SR162 é proveniente da mutação genética do cacau Catongo, que é caracterizado por possuir sementes brancas, e recebeu esse nome por ser oriundo da Fazenda São Roque, situada na cidade de Itagibá-BA. A cultivar PH16 foi identificada em uma população de cacaueiros híbridos da Fazenda Porto Híbrido, no município de São José da Vitória-BA, e a amostra de cacau convencional é composta por uma mistura de sementes sadias e doentes das variedades Pará, Parazinho e Maranhão.

As amêndoas fermentadas e secas foram recebidas após monitoramento das etapas de colheita, fermentação e secagem realizadas no mês de outubro/2010, na Fazenda Lajedo do Ouro, localizada no município de Ibirataia-Bahia. As amêndoas foram armazenadas em depósito com temperatura controlada e, em seguida, transportadas para a Fazenda Riachuelo, localizada na Estrada Ilhéus-Itabuna, Estado da Bahia, onde foram processadas de acordo com o fluxograma mostrado na Figura 1 e com a formulação apresentada na Tabela 1. 
Estudo reológico de chocolates elaborados com diferentes cultivares de cacau (Theobroma cacao L.) LEITE, P. B. et al.

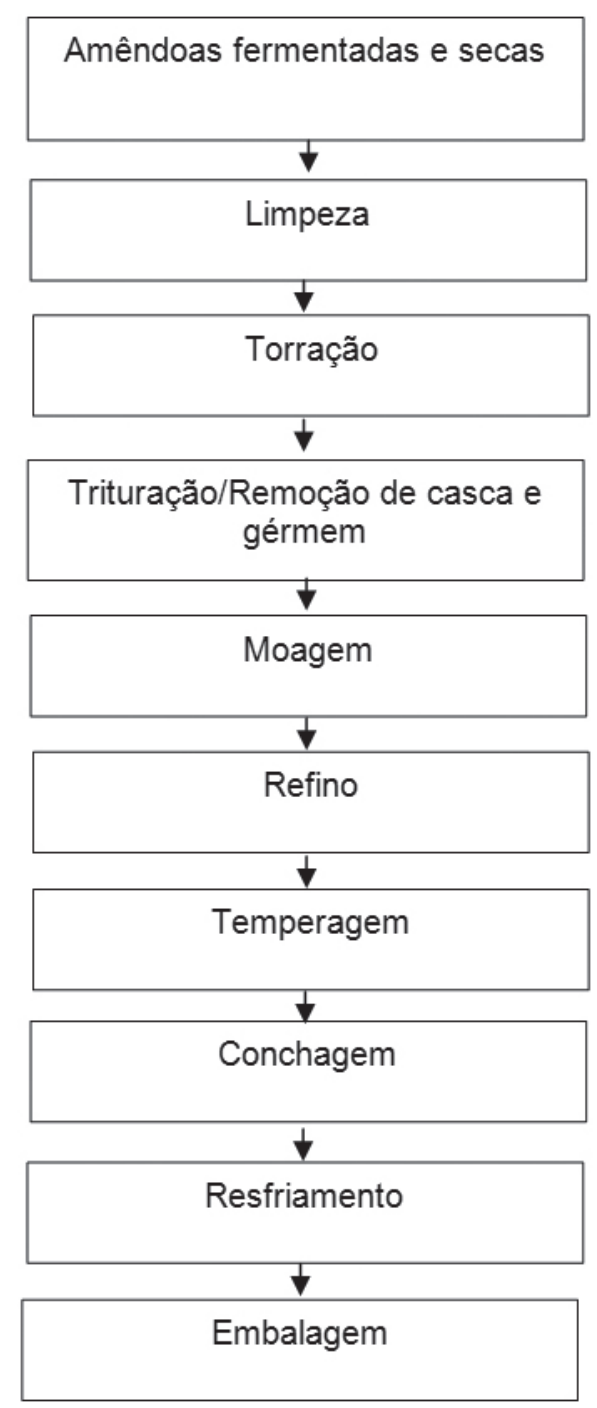

Figura 1. Fluxograma do processamento dos chocolates.

Tabela 1. Ingredientes das formulações dos chocolates.

\begin{tabular}{cc}
\hline Ingredientes & Quantidade (\%) \\
\hline Massa de cacau & 67,0 \\
Manteiga de cacau & 6,6 \\
Açúcar refinado & 26,0 \\
Lecitina de soja & 0,4 \\
\hline
\end{tabular}

\subsection{Processamento}

As amêndoas fermentadas e secas foram torradas em um torrador circular da marca Jaf Inox (São Paulo, Brasil), a uma temperatura de $120^{\circ} \mathrm{C}$, durante 2 horas. Em seguida, as amêndoas torradas foram trituradas em triturador da marca Jaff Inox (São Paulo, Brasil), sendo removidos a casca e o gérmen por fluxo de ar, originando o nibs de cacau. Os nibs foram triturados em moinho de facas, sendo que, nessa etapa, foi adicionado açúcar refinado comercial (marca União - Cia União S.A.). Posteriormente, a massa de cacau foi refinada em moinho de cinco rolos, obtendo-se uma granulometria ideal para o chocolate.

A massa refinada seguiu para a etapa de conchagem, realizada em concha horizontal da marca Jaf Inox (São Paulo, Brasil), à temperatura de $60^{\circ} \mathrm{C}$, por 48 horas. Nessa etapa, foram adicionadas a manteiga de cacau desodorizada comercial (JOANES Industrial S/A Produtos Químicos e Vegetais) e a lecitina de soja, também comercial (Bunge Alimentos S/A), contendo 55\% de fosfolipídios. O chocolate foi conduzido para o processo de temperagem, em temperadareira com mesa vibratória da marca Jaf Inox, até alcançar temperatura de $42^{\circ} \mathrm{C}$, para a formação de cristais estáveis da manteiga de cacau, e resfriado até atingir a temperatura de $28{ }^{\circ} \mathrm{C}$, sendo reaquecido para $31^{\circ} \mathrm{C}$, conforme Cunha et al. (2010); seguiu, então, para a moldagem em fôrma de polietileno, na forma de barras de 5 gramas e 1000 gramas, sendo posteriormente resfriado em freezer vertical e embalado, quando permaneceu à temperatura de $18^{\circ} \mathrm{C}$.

\subsection{Análises realizadas}

\subsubsection{Extração lipídica}

A extração e a determinação do teor de lipídeos foram feitas com tratamento ácido e posterior lavagem com água, seguidas de extração em Soxhlet. O ensaio foi feito em triplicata (SCHETTY et al., 1969; LANNES, 1997).

\subsubsection{Composição em triacilgliceróis}

As análises de triacilgliceróis foram realizadas em cromatógrafo a gás (modelo CGC, Agilent 6850 Series CG System, Santa Clara, USA). Foi utilizada coluna capilar (50\% fenilmetilpolisiloxane, $15 \mathrm{~m}$ de comprimento $\times 0,25$ mm de diâmetro interno) DB-17HT da marca Agilent (Santa Clara, CA, USA). As condições cromatográficas foram as seguintes: injeção split, taxa de 1:100, temperatura da coluna de $250{ }^{\circ} \mathrm{C}$, programada para elevar até $350^{\circ} \mathrm{C}$ a $5{ }^{\circ} \mathrm{C} / \mathrm{min}^{-1}$; gás de arraste: hélio, taxa de fluxo de 1.0 $\mathrm{mL}$. $\mathrm{min}^{-1}$; temperatura do injetor: $360^{\circ} \mathrm{C}$; temperatura do detetor: $375^{\circ} \mathrm{C}$; volume injetado: $1.0 \mu \mathrm{L}$; concentração da amostra: $100 \mathrm{mg} / 5 \mathrm{~mL}$ de hexano. A identificação dos grupos de triacilgliceróis foi realizada por comparação com os tempos de retenção (ANTONIOSI FILHO et al., 1995). A análise foi realizada em triplicata.

\subsubsection{Reologia}

Os ensaios foram realizados em um Reômetro MARS, Haake, à temperatura de $40^{\circ} \mathrm{C}$, segundo método da IOCCC (2000). Usou-se o sensor cone placa (C35/1 Ti polido), com gap de $0,024 \mathrm{~mm}$, ensaio rotacional com taxa controlada (CR), em três passos: (1) 0,00 1/s a 65,00 $1 / \mathrm{s}, \mathrm{t}=180 \mathrm{~s}$; (2) $65,001 / \mathrm{s}, \mathrm{t}=60 \mathrm{~s}$; (3) 65,00 1/s a 0,00 $1 / \mathrm{s}, \mathrm{t}=180 \mathrm{~s}$. A quantidade de amostra colocada sobre 
Estudo reológico de chocolates elaborados com diferentes cultivares de cacau (Theobroma cacao L.)

LEITE, P. B. et al.

a placa fixa do equipamento foi suficiente para cobrir uma área de aproximadamente $2 \mathrm{~cm}^{2}$. A quantidade de amostra também foi suficiente para preencher o espaço ente as placas. As análises foram realizadas em duplicata (IOCCC, 2000; LANNES, 2008).

\subsubsection{Determinação do tamanho máximo de partículas}

Micrômetro digital Digimatic Mitutoyo. Modelo Série 293. Determinação feita com auxílio de parafina, com cinco repetições, para verificação da repetibilidade.

\subsubsection{Tratamento estatístico dos dados}

Os dados foram submetidos à análise de variância (ANOVA) com subsequente teste de Tukey, em nível de significância de $p<0,05$, realizada com programa STATÍSTICA versão 7 (STATSOFT, 2004). O delineamento estatístico utilizado foi inteiramente casualizado.

\section{Resultados e discussão}

A Tabela 2 apresenta o conteúdo de gordura das três formulações de chocolates e a Tabela 3, a composição em triacilglicerois das gorduras extraídas das três formulações.

Praticamente, não se observou diferença na composição em triacilglicerois das três formulações, não sendo fator de influência para este estudo (Tabela 3).

Tabela 2. Conteúdo de gordura $\left(\mathrm{g} .100 \mathrm{~g}^{-1}\right)$ dos chocolates provenientes da amostra de cacau convencional e das cultivares de cacau SR162 e PH16.

\begin{tabular}{cccc} 
& & & \\
& Convencional & SR162 & PH16 \\
Média & $41,65^{a^{*}}$ & $41,76^{\mathrm{a}}$ & $36,53^{\mathrm{b}}$ \\
Desvio padrão & 0,14 & 0,13 & 0,16 \\
\hline
\end{tabular}

*Valores assinalados com a mesma letra na mesma linha entre as médias não diferem significativamente $(p>0,05)$, segundo o teste de Tukey $(n=3)$.

Tabela 3. Composição em triacilglicerois (média \pm desvio padrão) (g.100 $\mathrm{g}^{-1}$ ) das formulações de chocolates provenientes da amostra de cacau convencional e das cultivares de cacau SR162 e PH16.

\begin{tabular}{crrr} 
Triacilglicerol** $^{*}$ Convencional & SR162 & \multicolumn{1}{c}{ PH16 } \\
PoPP & $0,65 \pm 0,05^{a *}$ & $0,65 \pm 0,09^{a}$ & $0,66 \pm 0,03^{a}$ \\
PPP & $0,70 \pm 0,01^{a}$ & $0,75 \pm 0,06^{a}$ & $0,70 \pm 0,09^{a}$ \\
POP & $21,10 \pm 0,26^{a}$ & $20,34 \pm 0,27^{a}$ & $21,26 \pm 0,11^{a}$ \\
POO & $0,17 \pm 0,05^{a}$ & $0,21 \pm 0,02^{a}$ & $0,17 \pm 0,06^{a}$ \\
POS & $46,65 \pm 0,53^{a}$ & $46,30 \pm 0,41^{a}$ & $46,72 \pm 0,15^{a}$ \\
SOS & $29,60 \pm 0,30^{a}$ & $30,62 \pm 0,41^{a}$ & $29,46 \pm 0,20^{a}$ \\
SSS & $1,11 \pm 0,37^{a}$ & $1,15 \pm 0,42^{a}$ & $1,06 \pm 0,03^{a}$ \\
\hline
\end{tabular}

*Valores assinalados com a mesma letra na mesma linha entre as médias não diferem significativamente $(p>0,05)$, segundo 0 teste de Tukey $(n=3)$. ${ }^{\star *}$ PoPP, PPP, POP, POO, POS, SOS e SSS, sendo $\mathrm{Po}=$ palmitoleico, $\mathrm{P}=$ palmítico, $\mathrm{O}=$ oleico, $\mathrm{S}$ = esteárico.
A amostra proveniente da cultivar $\mathrm{PH} 16$, com menor conteúdo de gordura e maior tamanho de partículas, apresentou maior valor de tensão inicial e maior área de histerese, com mais ampla tixotropia (Figuras 2, 3 e 4), apontando a relação desses parâmetros com os itens mencionados. A viscosidade sofreu apenas pequeno aumento, não sendo essa diferença fator relevante na prática, apontando que tanto a variação da gordura como o tamanho das partículas não foram interferentes nesse parâmetro (Tabela 4).

Beckett (2000) explicou que o fenômeno da histerese não é uma surpresa, visto que a gordura extra só aumenta a movimentação livre das partículas, quando estas fluem no sistema. A maior quantidade das gorduras esta parcialmente ligada à superfície de partículas, tendo a gordura livre um grande efeito sobre o fluxo, diminuindo a viscosidade plástica. A tensão inicial é

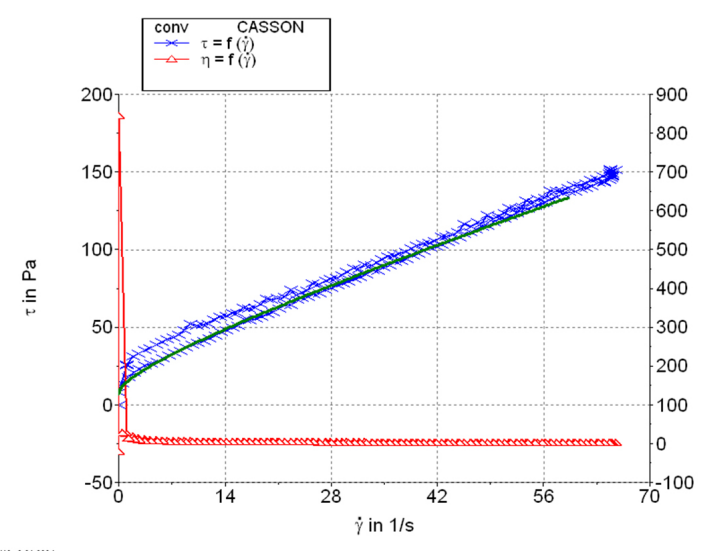

Figura 2. Curvas da análise reológica da amostra de chocolate proveniente da amostra de cacau convencional. T - tensão de cisalhamento (Pa), $\eta$ - viscosidade Casson (Pa.s), $\gamma$ - taxa de cisalhamento (1/s).

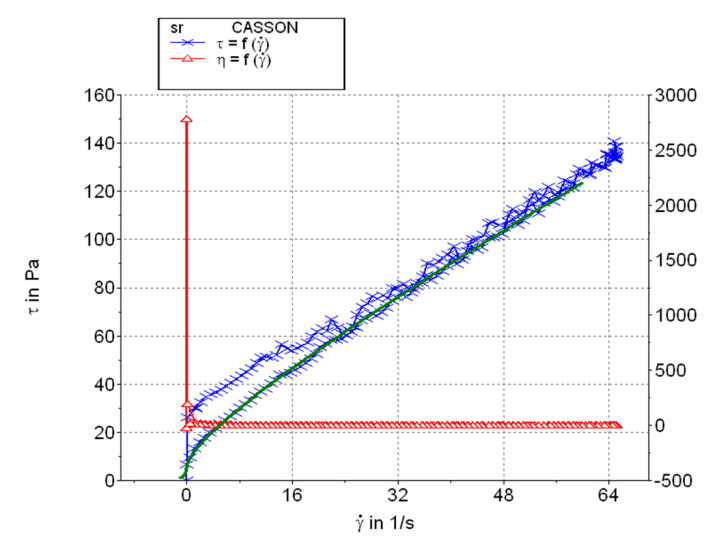

Figura 3. Curvas da análise reológica da amostra de chocolate proveniente da cultivar de cacau SR162. T - tensão de cisalhamento (Pa), $\eta$ - viscosidade Casson (Pa.s), $\gamma$ - taxa de cisalhamento (1/s). 


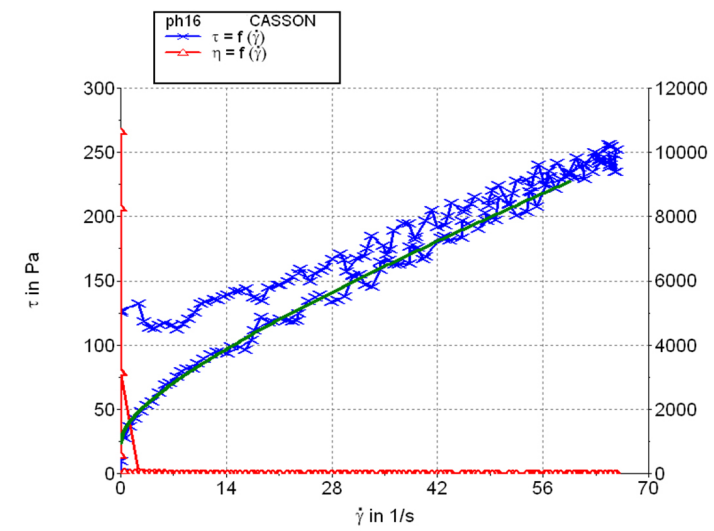

Figura 4. Curvas da análise reológica da amostra de chocolate proveniente da cultivar de cacau PH16. T - tensão de cisalhamento $(\mathrm{Pa}), \eta$ - viscosidade Casson (Pa.s), $\gamma$ - taxa de cisalhamento (1/s).

Tabela 4. Parâmetros de Casson das análises reológicas, tensão inicial de cisalhamento $\left(T_{c a}\right)$ e viscosidade plástica $\left(\eta_{c a}\right)$, e tamanho de partículas das amostras de chocolate (Media \pm Desvio pdrão).

\begin{tabular}{cccc} 
Amostra & $\mathrm{T}_{\mathrm{ca}}(\mathbf{P a})$ & $\eta_{\mathrm{ca}}$ (Pa.s) & $\begin{array}{c}\text { Tamanho } \\
\text { de partícula } \\
(\mu \mathrm{m})\end{array}$ \\
Convencional & $6,64 \pm 0,44^{\mathrm{a} *}$ & $1,63 \pm 0,44^{\mathrm{a}}$ & $17^{\mathrm{a}}$ \\
SR162 & $5,41 \pm 0,54^{\mathrm{a}}$ & $1,36 \pm 0,06^{\mathrm{a}}$ & $17^{\mathrm{a}}$ \\
PH16 & $20,91 \pm 4,37^{\mathrm{b}}$ & $1,79 \pm 0,07^{\mathrm{a}}$ & $21^{\mathrm{b}}$ \\
\hline
\end{tabular}

*Valores assinalados com a mesma letra na mesma coluna entre as médias não diferem significativamente $(p>0,05)$, segundo o teste de Tukey $(n=3)$.

mais pronunciada quando as forças de ligação entre as partículas sólidas são maiores, gerando menor efeito com adições de gordura.

Karnjanolarn e McCarthy (2006) estudaram a reologia de diferentes formulações de chocolate ao leite, variando as concentrações do emulsificante lecitina de soja. Na formulação contendo $0,4 \%$ do emulsificante, para moagem grossa (maior tamanho da partícula), o valor da tensão inicial de cisalhamento foi de 4,19 Pa e o da viscosidade plástica, de 1,63 Pa.s. Para o chocolate produzido com moagem fina (menor tamanho de partícula), a tensão inicial de cisalhamento foi de 10,4 Pa e a viscosidade plástica, de 2,26 Pa.s.

Vissotto et al. (1999) analisaram quatro marcas de chocolate meio amargo e encontraram, para a tensão de cisalhamento, valores de $7,11 \mathrm{~Pa}$ a $13,95 \mathrm{~Pa}$, e para a viscosidade plástica, valores entre 1,51 Pa.s e 3,34 Pa.s. Lannes et al. (2002) estudaram a reologia do 'chocolate' de cupuaçu e encontraram, para a formulação do chocolate meio amargo, a tensão de cisalhamento de 6,22 Pa e a viscoisidade plástica de 1,34 Pa.s, valores próximos aos encontrados para o chocolate elaborado a partir da amostra de cacau convencional no presente estudo.
Fernandes et al. (2013), na Venezuela, investigaram o comportamento reológico de amostras de chocolate contendo $60 \%, 70 \%$ e $75 \%$ de uma base de cacau (BC), constituída de 90\% de líquor de cacau e 10\% de manteiga de cacau. Os testes foram realizados em quatro temperaturas diferentes: $35^{\circ} \mathrm{C}, 40^{\circ} \mathrm{C}, 45^{\circ} \mathrm{C}$ e $50^{\circ} \mathrm{C}$. Na temperatura de $40^{\circ} \mathrm{C}$, os resultados para a tensão inicial de cisalhamento na amostra de chocolate contendo $60 \%$ da $\mathrm{BC}$ foi de 10,62 Pa; para a amostra contendo $70 \%$ da $\mathrm{BC}$, foi de $5,10 \mathrm{~Pa}$, e para a amostra contendo $75 \%$ da $\mathrm{BC}$, foi de $2,47 \mathrm{~Pa}$. Ainda na temperatura de $40{ }^{\circ} \mathrm{C}$, os resultados para a viscosidade plástica na amostra formulada com $60 \%$ da BC foi de 0,90 Pa.s; na amostra contendo $70 \%$, esse valor foi de 0,65 Pa.s e, para a amostra que continha $75 \%$ da BC, o valor encontrado foi 0,39 Pa.s. O chocolate elaborado com cacau proveniente da cultivar SR162 apresentou 73,6\% de cacau com o valor de 5,41 Pa para a tensão inicial de cisalhamento, valor próximo ao resultado encontrado pelos autores.

Cunha et al. (2010), avaliando o comportamento reológico de chocolate tipo meio amargo, variaram os teores de lecitina (entre 0,3 e 1,4\%) e poliglicerol poliricinolato (PGPR) na formulação, e concluíram que o comportamento da curva de viscosidade foi semelhante entre as amostras contendo apenas a lecitina e as amostras contendo lecitina e PGPR. Nas amostras de chocolate contendo concentrações acima de $0,6 \%$ de lecitina, o valor da viscosidade plástica manteve-se constante em cerca de 2,5 Pa.s. Neste trabalho, foi utilizada uma concentração de $0,4 \%$ de lecitina de soja, em que se bteve o resultado variando entre 1,36 e 1,79 Pa.s, valores menores ao encontrado pelos autores, resultante da menor concentração de lecitina empregada na formulação.

\section{Conclusões}

- A amostra com menor conteúdo de gordura e maior tamanho de partículas, $\mathrm{PH} 16$, apresentou maior valor de tensão inicial e maior área de histerese, com mais ampla tixotropia, apontando a relação desses parâmetros com os itens mencionados. Não foi encontrada diferença significativa nos valores de viscosidade aparente entre as amostras, indicando que a diferença no teor de manteiga de cacau presente e no tamanho máximo das partículas não influenciaram esse parâmetro reológico;

- Os resultados obtidos neste estudo mostraram que a viscosidade apresentada pelos chocolates produzidos não são unicamente dependentes do tamanho máximo das partículas e do teor de gordura, já que não houve diferença no perfil de triacilglicerois; 
Estudo reológico de chocolates elaborados com diferentes cultivares de cacau (Theobroma cacao L.) LEITE, P. B. et al.

- Como não houve diferenças significativas nas análises realizadas, entre as cultivares resistentes ao fungo e a cultivar tradicional, conclui-se que o processamento industrial dessas cultivares pode seguir o mesmo estabelecido para as cultivares tradicionais.

\section{Agradecimentos}

À FAPESB (Fundação de Amparo à Pesquisa do Estado da Bahia) e ao CNPq (Conselho Nacional de Desenvolvimento Científico e Tecnológico), pelo apoio financeiro.

\section{Referências}

ANTONIOSI FILHO, N.; MENDES, O. L.; LANÇAS, F. M. Computer prediction of triacylglycerol composition of vegetable oils by HRGC. Chromatographia, Wiesbaden, v. 40, p. 557-562, 1995. http://dx.doi.org/10.1007/BF02290268

BECKETT, S. T. The Science of Chocolate. London: Royal Society of Chemistry Paperbacks, 2000. 175 p.

BRASIL. Ministério da Saúde. Resolução de Diretoria Colegiada - RDC n²64, de 22 de setembro de 2005. Regulamento técnico para chocolate e produtos de cacau. Diário Oficial da República Federativa do Brasil, Brasília, DF, 23 set. 2005. Seção 1. Disponível em: <http://e-legis.anvisa. gov.br/leisref/public/showAct. php?id=18823\&word=>. Acesso em: 22 mar. 2011.

CUNHA, K. M.; QUAST, L. B.; LUCCAS, V. Influência da adição de lecitina de soja e dopoliglicerol poliricinolato nas propriedadesreológicas do chocolate. Boletim do CEPPA, Curitiba, v. 28, n. 2, p. 321-330, 2010.

GRAMACHO, I. C. P.; MAGNO, A. S. Cultivo e Beneficiamento do Cacau na Bahia. Ilhéus: CEPLAC/CEDEX, 1992. 124 p.

INTERNATIONAL COCOA ORGANIZATION - ICCO. Produção Mundial de cacau. London: ICCO, 2011. Disponível em: <http:// www.icco.org>. Acesso em: 16 out. 2011.

INTERNATIONAL OFFICE OF COCOA, CHOCOLATE AND SUGAR CONFECTIONERY - IOCCC. Viscosity of Cocoa and Chocolate Products. Geneva: International Office of Cocoa, Chocolate and Confectionary, 2000. Analytical method, 46-2000.

FERNANDES, V. A.; MÜLLER, A. J.; SANDOVAL, A. J. Thermal, structural and rheological characteristics of dark chocolate with different compositions. Journal of Food Engineering, Essex, v. 116, p. 97-108, 2013. http://dx.doi.org/10.1016/j. jfoodeng.2012.12.002

KARNJANOLARN, R.; McCARTHY, K. L. Rheology of different formlations of milk chocolate and the effect on coating thickness. Journal of Texture Studies, New York, v. 36, p. 668-680, 2006. http://dx.doi.org/10.1111/j.1745-4603.2006.00077.x

LANNES, S. C. S. Estudo das Propriedades Físico-químicas e de Textura de Chocolates. 1997. 175 f. Tese (Doutorado em Tecnologia Bioquímico - Farmacêutica)-Faculdade de Ciências Farmacêuticas, Universidade de São Paulo, São Paulo, 1997.

LANNES, S. C. S. Cupuaçu: uma Abordagem para sua Utilização como Substituto do Cacau. 2008. 144 f. Tese (Livre Docência)-Faculdade de Ciências Farmacêuticas, Universidade de São Paulo, São Paulo, 2008.

LANNES, S. C. S.; MEDEIROS, M. L.; AMARAL, R. L. Formulação de "chocolate" de cupuaçu e reologia do produto líquido. Brazilian Journal of Pharmaceutical Sciences, São Paulo, v. 38, n. 4, p. 463-469, 2002.

MÜLLER, H. G. Introducción a la Reología de los Alimentos. Zaragoza: Editorial Acribia, 1973. 174 p.

PEREIRA, J. L.; RAM, A.; FIGUEIREDO, J. M.; ALMEIDA, L. C. C. The First occurence of witches' broom disease in the principal cocoa region in Brasil. Tropical Agriculture, Trinidad e Tobago, v. 67, n. 2, p. 188-189, 1989.

PINTO, L. R. M.; PIRES, J.L. Seleção de Plantas de Cacau Resistentes à Vassoura-de-bruxa. Ilhéus: CEPLAC/CEPEC, 1998. 35 p. (Boletim Técnico, n. 181).

VISSOTTO, F. Z.; LUCCAS, V.; BRAGAGNOLO, N.; TUROTTI, J. M.; GRINALDI, R.; FIGUEIREDO, M. S. Caracterização físicoquímica e reológica de chocolates comerciais tipo cobertura elaborados comgorduras alternativas. Brazilian Journal Food Technology, Campinas, v. 2, n. 1,2, p. 139-148, 1999.

SCHETTY, O.; ANKER, P.; JUNKER, E.; KLEINERT, J. Schokolade. In: SCHEWEIZERISCHES lebensmittelbuch. 5. ed. [s.I.: s.n.], 1969. v. 3, cap. 36C, p. 47-51.

STATSOFT. Programa Computacional Statistica 7.0. Tulsa: Statsoft, 2004.

STEFFE, J. F. Rheological Methods in Food Process Engineering. 2nd ed. East Lansing: Freeman Press, 1996. 418 p. PMCid:PMC1303637. 